\title{
Double-Dose Pravastatin Versus Add-On Ezetimibe with Low-Dose Pravastatin - Effects on LDL Cholesterol, Cholesterol Absorption, and Cholesterol Synthesis in Japanese Patients with Hypercholest- erolemia (PEAS study)
}

\author{
Jun Sasaki ${ }^{1}$, Takatoshi Otonari ${ }^{2}$, Yasunori Sawayama ${ }^{3}$, Shiro Hata ${ }^{4}$, Yoshimi Oshima ${ }^{5}$, Tetsunori Saikawa ${ }^{6}$, \\ Sadatoshi Biro ${ }^{7}$, Suminori Kono ${ }^{3}$ for the PEAS study investigators. \\ ${ }^{1}$ International University of Health and Welfare, Graduate School of Pharmaceutical Medicine, Fukuoka, Japan \\ ${ }^{2}$ Otonari Clinic, Fukuoka, Japan \\ ${ }^{3}$ Kyushu University, Faculty of Medical Sciences, Fukuoka, Japan \\ ${ }^{4} \mathrm{NHO}$ Ureshino Medical Center, Saga, Japan \\ ${ }^{5}$ Oshima Clinic, Oita, Japan \\ ${ }^{6}$ Oita University, Faculty of Medicine, Oita, Japan \\ ${ }^{7}$ Tsukasa Health Care Hospital, Kagoshima, Japan
}

\begin{abstract}
Aim: This study compared the effect of doubling the dose of pravastatin with that of adding ezetimibe to low-dose pravastatin on the LDL cholesterol (LDL-C) level and on cholesterol absorption and synthesis markers. The tolerability of the 2 regimens was also compared.

Methods: This was a multicenter, open-label, parallel-group trial. Subjects were aged from 20 to 74 years and had an LDL-C $\geq 120 \mathrm{mg} / \mathrm{dL}$ despite pravastatin therapy at $5-10 \mathrm{mg} /$ day. They were randomly allocated to receive either add-on ezetimibe $(10 \mathrm{mg} /$ day $)$ or double-dose pravastatin, and follow-up was performed for 12 weeks. The primary endpoints were the changes of LDL-C and apolipoprotein (apo) B levels after 12 weeks of treatment. Cholesterol absorption and synthesis markers were also determined.

Results: LDL-C and apo B decreased by $16 \%$ and $14 \%$ in the ezetimibe add-on group versus $5.9 \%$ and $4.4 \%$, respectively, in the pravastatin double-dose group. The between-group differences of these decreases were highly significant. Cholesterol absorption markers (sitosterol, campesterol, and cholestanol) were reduced by $48 \%, 36 \%$, and $10 \%$, respectively, in the ezetimibe add-on group, and were increased by $17 \%, 14 \%$, and $6 \%$, respectively, in the pravastatin double-dose group. Lathosterol (a cholesterol synthesis marker) increased by $76 \%$ in the ezetimibe add-on group and by $24 \%$ in the pravastatin double-dose group. The difference was statistically significant. No serious adverse effect was observed in either group.

Conclusions: Adding ezetimibe to low-dose pravastatin achieves greater decreases in LDL-C, apo B, and cholesterol absorption markers than doubling the dose of pravastatin.
\end{abstract}

J Atheroscler Thromb, 2012; 19:485-493.

Key words; Cholesterol, Absorption, Synthesis, Pravastatin, Ezetimibe

\section{Introduction}

An elevated blood level of cholesterol is a major

Address for correspondence: Jun Sasaki, International University of Health and Welfare, Graduate School of Pharmaceutical Medicine, 1-3-1, Nagahama, Chuo-ku, Fukuoka 810-0072, Japan E-mail: jsas@nifty.com

Received: October 4, 2011

Accepted for publication: November 22, 2011 risk factor for cerebrovascular and cardiovascular diseases. Cholesterol is supplied by two sources, i.e., synthesis in the liver and absorption from the small intestine. Currently, statin, a HMG-CoA reductase inhibitor, therapy targeting the inhibition of hepatic cholesterol synthesis is the mainstay for reducing low-density lipoprotein cholesterol (LDL-C), with a decrease of overall mortality being confirmed by meta-analysis ${ }^{1)}$. Subgroup analysis of the Scandinavian Simvastatin 
Survival Study (4S), a secondary prevention trial using simvastatin, revealed that statin therapy was effective for preventing coronary events in patients with low dietary cholesterol absorption, whereas the efficacy of statins was not sufficient in patients with increased cholesterol absorption ${ }^{2)}$. The Drugs and Evidencebased Medicine in the Elderly (DEBATE) study prospectively examined the relation between cholesterol absorption and cerebrovascular and cardiovascular events, and demonstrated that total mortality and the risk of cerebrovascular/cardiovascular events were higher in patients with increased cholesterol absorption than in those without an increase, even though LDL-C levels were similar ${ }^{3)}$. In other words, for LDLC-lowering therapy to achieve more efficient suppression of cerebrovascular/cardiovascular events, inhibition of cholesterol absorption from the small intestine is required, in addition to inhibition of hepatic cholesterol synthesis.

Ezetimibe is a selective inhibitor of the cholesterol transporter Niemann Pick C1 Like 1 (NPC1L1) expressed in the brush border membrane of the small intestine. This has expanded our treatment options, making it possible to improve the management of hypercholesterolemia.

Doubling the dose of a statin only achieves a small additional LDL-C-lowering effect (about 6\%), probably due to a compensatory increase of cholesterol absorption ${ }^{4}$. Because there is an inverse correlation between the LDL-C-lowering effect of the cholesterol absorption inhibitor ezetimibe and statins ${ }^{5)}$, it is possible that patients who do not achieve a sufficient reduction in LDL-C concentrations in response to statin monotherapy may benefit from additional treatment with ezetimibe to inhibit cholesterol absorption; however, the effectiveness of low-dose pravastatin combined with ezetimibe has not been established. We investigated the effects of adding ezetimibe on serum lipids and cholesterol absorption and synthesis in patients with high LDL-C levels who had not shown a sufficient response to low-dose pravastatin (5-10 $\mathrm{mg}$ /day), a standard treatment in Japan, by comparing the effects of doubling the pravastatin dose.

\section{Patients and Methods}

\section{Patients}

The subjects were outpatients aged 20 to 74 years who had LDL-C $\geq 120 \mathrm{mg} / \mathrm{dL}$ after treatment with pravastatin ( $5 \mathrm{mg}$ or $10 \mathrm{mg}$ per day) for 4 weeks or longer. LDL-C $<120 \mathrm{mg} / \mathrm{dL}$ is the target of cholesterol-lowering therapy for high-risk patients without coronary artery disease according to the Japan Athero- sclerosis Society ${ }^{6}$. The exclusion criteria were use of a drug or drugs for hyperlipidemia other than the study drugs, insulin, or thiazolidinediones; a history of hypersensitivity to the study drugs, severe liver or kidney diseases; acute coronary syndrome (myocardial infarction and unstable angina) or stroke in the past 3 months, familial hypercholesterolemia or secondary hyperlipidemia; pregnancy including possible pregnancy or breast-feeding; and use of fats/oils enriched with plant sterols. Patients could also be ineligible if the study physician considered that participation was inappropriate.

\section{Study Design}

Eligible patients were enrolled at the registration center and were randomly allocated to either the ezetimibe add-on group or pravastatin double-dose group. The ezetimibe group received ezetimibe $10 \mathrm{mg} /$ day as add-on therapy, and the pravastatin group started a doubled dose of pravastatin. The study period was 12 weeks. The patients visited their study physician every 4 weeks. Drugs other than those specified above were allowed, but study physicians were asked to maintain the dose during the study period. The study was approved by the Ethics Committee of the International University of Health and Welfare. Written informed consent was obtained from all the participants.

Recorded at the beginning of the study were height $(\mathrm{cm})$, smoking and alcohol use, comorbid conditions, and concomitant medications. Serum lipids and lipoproteins, markers of cholesterol absorption and synthesis, and other biochemical items, as well as weight $(\mathrm{kg})$ and waist circumference $(\mathrm{cm})$, were measured at the beginning of the study and after 12 weeks of treatment. Changes of medications and adverse events were recorded during the course of the study. Adherence was assessed on the basis of self-reported compliance in the past 4 weeks assessed at week 4,8 and 12 each. Frequency of use of the prescribed drug was elicited by a closed-ended question per week (daily; 5-6, 3-4 or 1-2 days per week; and null per week), and the average of the three frequencies was obtained as global compliance.

The primary outcome variables were the percent changes of LDL-C, apolipoprotein (apo B) and markers of cholesterol absorption and synthesis. The secondary outcome variables were serum lipids other than LDL-C, glucose metabolism parameters, C-reactive protein (CRP), and adiponectin.

Tolerability was assessed at each study visit and included adverse events, physical examination findings, and clinical laboratory test results. Abnormal 
laboratory test results were defined as $>3$ times of the upper limit of normal for alanine aminotranferase (ALT) or aspartate aminotranferase (AST), 10 times the upper limit of normal for creatine kinase $(\mathrm{CK})$, or 1.5 times the upper limit of normal for creatinine.

\section{Laboratory Assays}

All laboratory measurements were conducted at an external laboratory (SRL, Tokyo). Serum total cholesterol (TC) and triglycerides (TG) were measured by enzymatic methods, and high-density lipoprotein cholesterol (HDL-C) and LDL-C were measured by direct methods using commercial reagents (Sekisui Medical Co. Ltd., Tokyo). Apo AI, apo B, and apo E were measured by an immunoturbidimetric method. Fasting blood glucose was measured by the hexokinase method, HbA1c by the latex agglutination immunoassay, and fasting insulin by chemiluminescent enzyme immunoassay. Homeostasis model assessment of insulin resistance index (HOMA-IR) was calculated by dividing the product of fasting plasma glucose $(\mathrm{mg} /$ $\mathrm{dL}$ ) and fasting serum insulin (IU/L) by 405 .

Cholesterol absorption was assessed by sitosterol, campesterol, and cholestanol; sitosterol, campesterol are plant sterols that are not synthesized de novo, and cholestanol is a bile acid metabolite. Lathosterol, an intermediate metabolite in the cholesterol-biosynthesis pathway, was used as a marker of cholesterol synthesis. These cholesterol absorption and synthesis markers were determined by gas chromatography according to the method of Miettinen et al. ${ }^{4)}$. Sterol measurements below the detection limit, $1.0 \mu \mathrm{g} / \mathrm{mL}$, were assigned a value of $0.5 \mu \mathrm{g} / \mathrm{mL}$.

High-sensitivity CRP (hs-CRP) was measured by immunonephelometry, and high molecular weight adiponectin was measured by ELISA (Sekisui Medical Co. Ltd., Tokyo). CRP $>10 \mathrm{mg} / \mathrm{mL}$ was discarded because acute inflammation was suspected ${ }^{7}$.

The detection limit of CRP concentrations was $0.05 \mathrm{mg} / \mathrm{L}$, and undetectable values were recoded as $0.025 \mathrm{mg} / \mathrm{L}$. AST, ALT and CK were measured by the Japanese Society of Clinical Chemistry standard methods, and serum creatine was determined by enzymatic methods.

\section{Statistical Analysis}

Mean and SD are presented for continuous variables. Between-group and within-group comparisons were statistically tested by the Wilcoxon rank-sum test (Mann-Whitney $U$ test) and Wilcoxon signed-rank test. Analysis of covariance was used when adjustment was needed for differences at the baseline. Fisher's exact test was used for comparison of categorical vari- ables at the baseline. $P<0.05$ was considered significant.

As for markers of cholesterol absorption and synthesis, limited information was available to assess the expected magnitude of the between-group difference and variance of the percent change; therefore, we arbitrarily decided that the sample size should be 100 patients for each group (200 subjects).

\section{Results}

\section{Study Population}

A total of 198 patients were enrolled in the study, and 101 and 97 patients were randomly assigned to the ezetimibe add-on group and pravastatin doubledose group, respectively. Except for one patient in the latter group who withdrew before the start of treatment, 197 patients started the treatment as assigned. Of these, 191 patients $(n=96$ in the ezetimibe add-on group and $n=95$ in the pravastatin double-dose group) completed a 12-week study (Fig. 1). The efficacy analysis population was composed of these 191 patients. Three patients in the pravastatin group were found to have used pioglitazone through the study period. The population for tolerability evaluation consisted of all the patients who started the treatment, including those who ceased the treatment due to adverse events $(n=3)$, did not visit after 4 weeks $(n=1)$, had no blood sampling at 12 weeks $(n=1)$, were treated with the opposite modality $(n=1)$; the patient in the last category also had no blood sampling at 12 weeks.

There was no difference between the two groups in terms of subject characteristics, except for a higher prescription of anti-diabetic drug in the pravastatin double-dose group (Table 1). The ezetimibe add-on group had higher concentrations of TC, non-HDL-C, LDL-C, apo B, and fasting serum insulin than the pravastatin double-dose group (Table 2).

\section{Efficacy}

Table 3 summarizes the percent changes of lipids and apolipoproteins, glucose metabolism parameters, CRP, and adiponectin after 12 -week treatment. TC, non-HDL-C, LDL-C, and apo B concentrations were decreased significantly in both groups, but the decreases were more marked in the ezetimibe add-on group showing significant between-group differences. Add-on ezetimibe resulted in a $16 \%$ decrease in LDL-C while no more than $6 \%$ decrease in LDL-C was observed in the double-dose pravastatin treatment. TG increased significantly in the pravastatin double-dose group only, and apo E decreased signifi- 


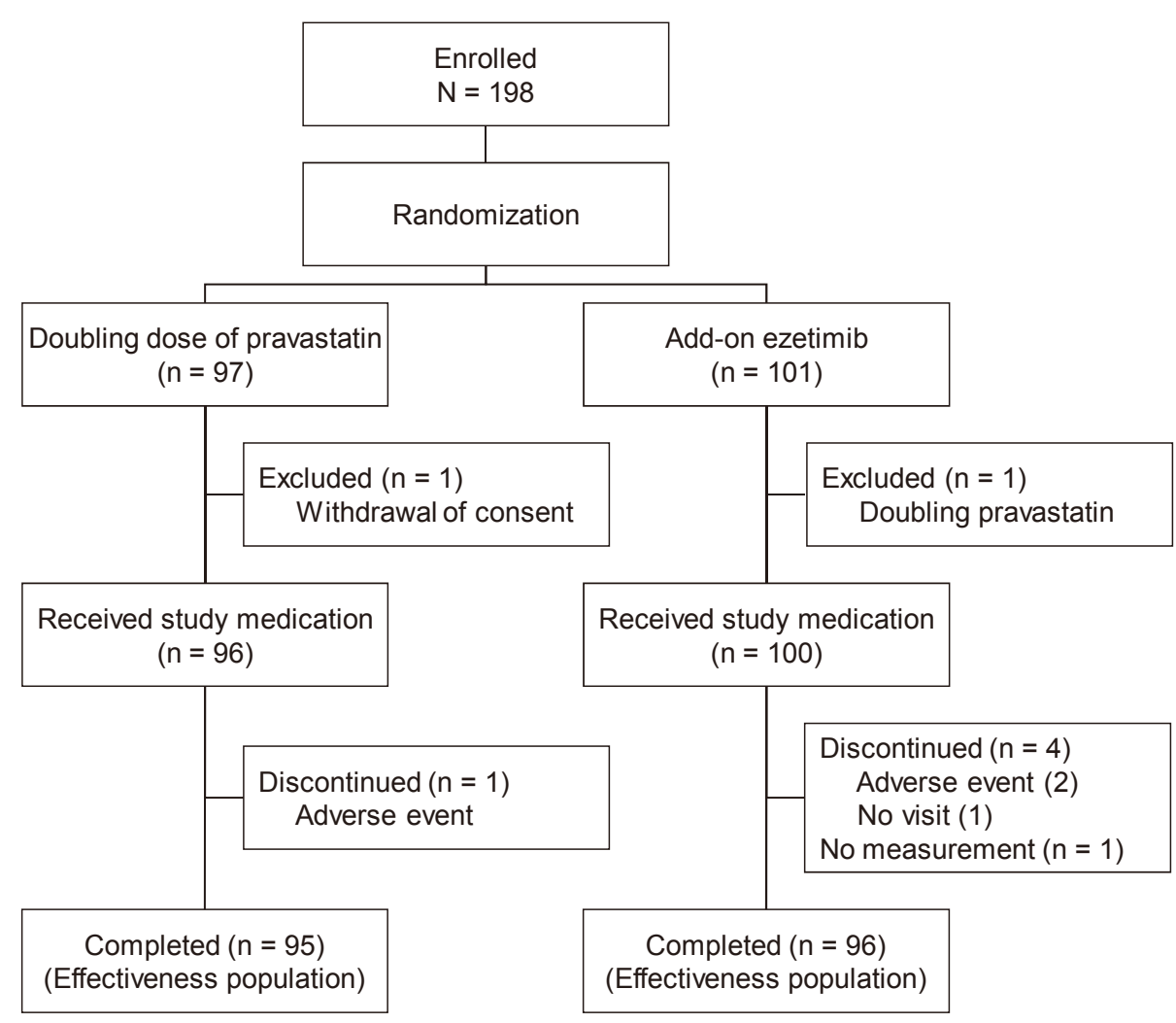

Fig. 1. Disposition of the subjects.

cantly in the ezetimibe add-on group only. The between-group difference was significant for the percent change of TG and apo E.

Fasting glucose and HbAlc did not change after either treatment, but fasting insulin and HOMA-IR increased in both groups, the increases being significant in the pravastatin double-dose group. There was no effect of the treatment on CRP and adiponectin concentrations. Waist circumference showed a statistically significant decrease in the ezetimibe add-on group, and the decrease was significantly different from that of the pravastatin double-dose group. Decreases in body weight showed no difference between the two groups (Table 3).

Because the baseline levels of TC, non-HDL-C, LDL-C, and apo B were higher in the ezetimibe group, we performed analysis of covariance to adjust for the baseline concentrations. The between-group differences in the percent changes of TC, non-HDL-C, LDL-C, and apo B were almost the same as those unadjusted for the baseline concentrations. Adjusted means of the percent decrease in LDL-C were 14.4\% (95\% confidence interval $[\mathrm{CI}] 12.0$ to 18.2$)$ in the ezetimibe add-on group and $7.2 \%$ (95\% CI 4.0 to 10.4 ) in the pravastatin double-dose group. The corresponding values for apo B were 13.1 (95\% CI 10.4 to 15.9 ) and $5.2 \%$ (95\% CI 2.5 to 8.0$)$.

The markers of cholesterol absorption decreased substantially in the ezetimibe add-on group (Table 4). The percent decreases of ratios of absorption markers to cholesterol were also remarkable, i.e., $48 \%$ for campesterol, $36 \%$ for sitosterol, and $10 \%$ for cholestanol. On the other hand, these absorption markers increased in the pravastatin double-dose group; the percent increases of the ratios to cholesterol were $17 \%$ for campesterol, $14 \%$ for sitosterol, and $6 \%$ for cholestanol. In contrast, lathosterol (a marker of cholesterol synthesis) increased in both groups, but more markedly in the ezetimibe add-on group. The increase of lathosterol adjusted for total cholesterol concentrations was $76 \%$ in the ezetimibe add-on group and $24 \%$ in the pravastatin double-dose group.

\section{Tolerability}

The overall compliance was estimated for 73 patients in the ezetimibe add-on group and 74 in the pravastatin double-dose group. On average, the prescribed drug was taken 6.8 days per week in the former and 6.9 days per week in the latter.

Adverse effects numbered 18 among 17 patients 
Table 1. Characteristics of the study subjects at baseline

\begin{tabular}{|c|c|c|c|}
\hline Characteristic & $\begin{array}{l}\text { Pravastatin + ezetimibe } \\
\qquad(n=96)\end{array}$ & $\begin{array}{l}\text { Double-dose pravastatin } \\
\qquad(n=95)\end{array}$ & $p$ value ${ }^{*}$ \\
\hline Female, n (\%) & $63(65.6)$ & $69(72.6)$ & 0.35 \\
\hline Age (year), mean (SD) & $66.3(9.7)$ & $69.0(9.6)$ & 0.05 \\
\hline Smoking, n (\%) & $15(15.6)$ & $8(8.4)$ & 0.18 \\
\hline Regular alcohol use, n (\%) & $27(28.1)$ & $17(17.9)$ & 0.12 \\
\hline Body weight (kg), mean (SD) & $58.7(10.5)$ & $57.9(10.8)$ & 0.54 \\
\hline BMI $\left(\mathrm{kg} / \mathrm{m}^{2}\right)$, mean $(\mathrm{SD})$ & $24.2(3.5)$ & $24.1(3.6)$ & 0.67 \\
\hline Waist $(\mathrm{cm})$, mean $(\mathrm{SD})$ & $87.5(8.0)$ & $88.0(8.3)$ & 0.87 \\
\hline \multicolumn{4}{|l|}{ Comorbidity } \\
\hline Liver diseases, n (\%) & $3(3.1)$ & $2(2.1)$ & 1.00 \\
\hline Hypertension, n (\%) & $64(66.7)$ & $69(72.6)$ & 0.43 \\
\hline Antihypertensive drug, n (\%) & $55(57.3)$ & $61(64.2)$ & 0.37 \\
\hline Diabetes/IGT, n (\%) & $35(36.5)$ & $40(42.1)$ & 0.46 \\
\hline Antidiabetic drug, n (\%) & $9(9.4)$ & $19(20.0)$ & 0.04 \\
\hline $\mathrm{CAD}, \mathrm{n}(\%)$ & $8(8.3)$ & $16(16.8)$ & 0.08 \\
\hline \multicolumn{4}{|l|}{ Prior history } \\
\hline Myocardial infarction, n (\%) & $1(1.0)$ & $4(4.2)$ & 0.21 \\
\hline Cerebral infarction, n (\%) & $14(14.6)$ & $8(8.4)$ & 0.26 \\
\hline Parental CAD, n (\%) & $35(36.5)$ & $28(29.5)$ & 0.36 \\
\hline Pravastatin 10 mg, n (\%) & $30(31.3)$ & $29(30.5)$ & 1.00 \\
\hline
\end{tabular}

$\mathrm{CAD}=$ coronary artery diseases; IGT $=$ impaired glucose tolerance

* Based on Fisher's exact test for proportions and Wilcoxon rank-sum test for continuous variables.

in the ezetimibe add-on group and 10 among 7 patients in the pravastatin double-dose group. Of these, 3 cases (diarrhea, rash, and insomnia) in the ezetimibe add-on group and 2 cases (leg cramps and mild liver dysfunction) in the pravastatin double-dose group were considered by study physicians to be probably and possibly related to the study drug, respectively. Rather serious cases of adverse effects, to which the trial drug was unrelated, were ankle sprain with an episode of abnormal elevation of CK from excessive exercise in the ezetimibe add-on group and fracture of the femoral neck in the pravastatin double-dose group. None had an episode of abnormal elevation of AST, ALT, or creatinine as specified a priori.

\section{Discussion}

Compared with doubling the dose of pravastatin, add-on ezetimibe resulted in greater decreases in LDL-C and apo B concentrations in patients under treatment with a low-dose pravastatin. The add-on ezetimibe also produced substantial decreases in cholesterol-absorption markers and an increase in a cholesterol-synthesis marker.

Several studies have examined the effect of addon ezetimibe therapy in patients under statin treat- ment. Among patients on basal statin treatment, addon ezetimibe resulted in a $21 \%$ to $27 \%$ additional reduction in LDL-C compared to add-on placebo ${ }^{8-10)}$. In the EASE study, which included 3,030 patients under statin treatment who had failed to attain the LDL-C target set by the National Cholesterol Education Program Adult Treatment Panel III, a 23\% reduction of LDL-C was obtained in the add-on ezetimibe group compared with the add-on placebo group ${ }^{11}$. Furthermore, in a pooled analysis of 4 studies $(2,382$ patients) that compared the effects of statin monotherapy and combination therapy of statin and ezetimibe, the percent decrease of LDL-C was 33\% in the former and $47 \%$ in the latter ${ }^{12}$.

In a study that investigated the combination use of pravastatin $(10,20$, or $40 \mathrm{mg} /$ day $)$ and ezetimibe $(10 \mathrm{mg} /$ day) for 12 weeks, pravastatin plus ezetimibe resulted in an average 39\% decrease of LDL-C while pravastatin alone decreased LDL-C by $25 \%$ on average $^{13)}$. The decreases in LDL-C were dependent on the pravastatin dose, but further decreases in LDL-C by the addition of ezetimibe were almost consistent across pravastatin doses (12-14\%). The combination of pravastatin $(10 \mathrm{mg} /$ day $)$ and ezetimibe $(10 \mathrm{mg} /$ day $)$ decreased LDL-C by 34\% while the decreases by 20 $\mathrm{mg} /$ day and $40 \mathrm{mg} /$ day of pravastatin alone were $24 \%$ 
Table 2. Serum lipids and lipoproteins and glucose metabolism parameters at baseline

\begin{tabular}{|c|c|c|c|c|c|}
\hline \multirow{2}{*}{ Variable (unit) } & \multicolumn{2}{|c|}{ Pravastatin + ezetimibe } & \multicolumn{2}{|c|}{ Double-dose pravastatin } & \multirow{2}{*}{$p$ value } \\
\hline & $n$ & Mean (SD) & $n$ & Mean (SD) & \\
\hline Total cholesterol (mg/dL) & 96 & $228.4(26.9)$ & 95 & $218.7(29.5)$ & 0.008 \\
\hline Non-HDL-C (mg/dL) & 96 & $171.5(26.6)$ & 95 & $161.6(27.5)$ & 0.003 \\
\hline LDL-C (mg/dL) & 96 & $142.5(21.5)$ & 95 & $133.0(23.1)$ & 0.002 \\
\hline HDL-C (mg/dL) & 96 & $56.9(12.4)$ & 95 & $57.1(13.6)$ & 0.96 \\
\hline $\mathrm{TG}(\mathrm{mg} / \mathrm{dL})$ & 91 & $133.7(62.1)$ & 89 & $123.8(71.0)$ & 0.09 \\
\hline Apo A (mg/dL) & 96 & $147.4(23.2)$ & 95 & $145.1(26.2)$ & 0.36 \\
\hline Apo B (mg/dL) & 96 & $115.8(17.8)$ & 95 & $109.0(18.2)$ & 0.005 \\
\hline Apo E (mg/dL) & 96 & $4.65(1.09)$ & 95 & $4.61(1.35)$ & 0.63 \\
\hline Fasting glucose (mg/dL) & 91 & $97.3(12.4)$ & 89 & $101.1(19.6)$ & 0.50 \\
\hline Fasting insulin $(\mu \mathrm{U} / \mathrm{mL})$ & 91 & $6.38(3.82)$ & 89 & $5.35(2.71)$ & 0.07 \\
\hline $\mathrm{HOMA}^{-\mathrm{IR}^{\dagger}}$ & 91 & $1.56(1.00)$ & 89 & $1.37(0.83)$ & 0.21 \\
\hline HbA1c (\%) & 96 & $5.61(0.51)$ & 95 & $5.77(0.79)$ & 0.42 \\
\hline hs-CRP (mg/L) & 95 & $1.04(1.54)$ & 92 & $0.93(1.23)$ & 0.78 \\
\hline Adiponectin $(\mu \mathrm{g} / \mathrm{mL})^{\dagger}$ & 94 & $6.94(4.78)$ & 95 & $8.00(4.44)$ & 0.04 \\
\hline
\end{tabular}

HDL-C = high-density lipoprotein cholesterol; LDL-C=low-density lipoprotein cholesterol; Apo=apolipoprotein; HOMA-IR= homeostasis model assessment of insulin resistance index; hs-CRP=high-sensitivity C-reactive protein.

${ }^{\dagger}$ High-molecular weight adiponectin.

and $29 \%$, respectively ${ }^{13)}$. Another study also showed that a combination of pravastatin $(10 \mathrm{mg} /$ day $)$ and ezetimibe $(10 \mathrm{mg} /$ day $)$ produced a greater decrease in LDL-C than a single treatment with pravastatin of 40 $\mathrm{mg} /$ day $(26 \% \text { versus } 20 \%)^{14)}$. In the present study, 10 $\mathrm{mg} /$ day of ezetimibe added to low-dose pravastatin (5-10 $\mathrm{mg} /$ day) decreased LDL-C by $16 \%$ while doubling the dose of pravastatin resulted in only a $6 \%$ decrease in LDL-C. The observed magnitude of the incremental decrease in LDL-C resulted by ezetimibe $(10 \mathrm{mg} /$ day $)$ is very similar to that reported for combination therapy ${ }^{13,14)}$.

The ezetimibe add-on decreased cholesterolabsorption markers and increased a cholesterol-synthesis marker. The present findings corroborate the previous observation in terms of the magnitude of the changes in the markers. It was reported that 2-week treatment with ezetimibe $10 \mathrm{mg} /$ day resulted in a $41 \%$ decrease in the campesterol/cholesterol ratio (an indicator of cholesterol absorption) and a $72 \%$ increase in the lathosterol/cholesterol ratio (an indicator of cholesterol synthesis) ${ }^{15}$. A unique finding in the present study was that similar changes were observed after administration of ezetimibe in addition to pravastatin. In the ezetimibe add-on group, the campesterol/cholesterol ratio and sitosterol/cholesterol ratio decreased by $48 \%$ and $36 \%$, respectively, and the lathosterol/ cholesterol ratio increased by $76 \%$. It is hypothesized that inhibition of cholesterol absorption by ezetimibe leads to a compensatory increase of cholesterol synthesis ${ }^{14)}$, but the increase of cholesterol synthesis by ezetimibe does not deteriorate LDL-C concentrations. A previous report suggested that ezetimibe increased the serum level of lathosterol while coadministration of ezetimibe and statin increased the fractional catabolic rate of apo $\mathrm{B}$ containing lipoproteins ${ }^{16)}$. In the present study, rather surprisingly, a double dose of pravastatin increased a cholesterol synthesis marker, although the increase was modest. In the double-dose pravastatin group, 13 patients showed a more than 2 -fold increase in the lathosterol/cholesterol ratio. Even after exclusion of these patients, there was no indication of a decrease in the cholesterol synthesis marker associated with double-dose pravastatin; mean percent change of the lathosterol/cholesterol ratio was $-0.4 \%$ (SD 34.1\%, $p=0.95$ ).

As confirmed in the present study, doubling the dose of a statin conferred only a small, additional effect and LDL-C could be further decreased by $5-6 \%{ }^{17)}$ In the Pravastatin or Atorvastatin Evaluation and Infection Therapy (PROVE-IT) study ${ }^{18)}$, LDL-C increased gradually at later periods of long-term treatment with standard (pravastatin $40 \mathrm{mg}$ /day) or intensive (atorvastatin $80 \mathrm{mg} /$ day) therapy. A compensatory increase in cholesterol absorption secondary to the inhibition of cholesterol synthesis by statin is the most likely explanation for the so-called escape phenomenon ${ }^{17,18)}$. Addition of ezetimibe to statin monotherapy 
Table 3. Percent changes in serum lipids and apolipoproteins and glucose metabolism parameters after 12 -week treatment

\begin{tabular}{|c|c|c|c|c|c|c|c|}
\hline Variable & \multicolumn{3}{|c|}{ Pravastatin + ezetimibe } & \multicolumn{3}{|c|}{ Double-dose pravastatin } & $p$ value $^{\dagger}$ \\
\hline Body weight & 96 & $-0.3(1.6)$ & 0.02 & 95 & $-0.4(2.1)$ & 0.02 & 0.72 \\
\hline Waist circumference & 96 & $-1.1(2.3)$ & $<0.0001$ & 95 & $-0.4(2.3)$ & 0.10 & 0.02 \\
\hline Total cholesterol & 96 & $-11.1(11.7)$ & $<0.0001$ & 95 & $-3.6(13.9)$ & 0.0004 & $<0.0001$ \\
\hline Non-HDL-C & 96 & $-16.1(14.1)$ & $<0.0001$ & 95 & $-5.0(17.7)$ & 0.0002 & $<0.0001$ \\
\hline TG & 84 & $-4.8(29.3)$ & 0.06 & 87 & $17.4(47.3)$ & 0.01 & 0.002 \\
\hline Apo A & 96 & $4.1(10.8)$ & 0.0005 & 95 & $4.3(13.0)$ & 0.002 & 0.71 \\
\hline Apo B & 96 & $-13.9(13.2)$ & $<0.0001$ & 95 & $-4.4(14.9)$ & 0.0007 & $<0.0001$ \\
\hline Apo E & 96 & $-5.9(12.4)$ & $<0.0001$ & 95 & $1.9(14.8)$ & 0.88 & 0.0002 \\
\hline Fasting glucose & 84 & $0.5(7.5)$ & 0.82 & 87 & $1.7(9.3)$ & 0.10 & 0.32 \\
\hline hs-CRP & 94 & $28.3(134)$ & 0.82 & 90 & $25.1(133)$ & 0.83 & 0.996 \\
\hline Adiponectin ${ }^{*}$ & 94 & $3.4(27.1)$ & 0.88 & 95 & $7.0(44.1)$ & 0.30 & 0.58 \\
\hline
\end{tabular}

HDL-C = high-density lipoprotein cholesterol; LDL-C = low-density lipoprotein cholesterol; Apo = apolipoprotein; HOMA-IR=homeostasis model assessment of insulin resistance index; hs-CRP=high-sensitivity C-reactive protein.

*Within-group comparison for difference from the baseline.

Between-group comparison.

${ }^{*}$ High-molecular weight adiponectin

selectively inhibits intestinal cholesterol absorption, thereby conferring a greater LDL-lowering effect than a higher dose of statin.

The waist circumference decreased after the addition of ezetimibe, but not after doubling the dose of pravastatin. In obesity-model rats, ezetimibe not only improved blood levels of chylomicrons and fatty acids but also suppressed the expression of sterol regulation element binding protein-1c (SREBP-1c), a transcription factor involved in hepatic fatty acid synthesis ${ }^{19)}$. Two-month administration of ezetimibe $10 \mathrm{mg} /$ day markedly inhibited postprandial increases of apo B48, a dominant apolipoprotein almost exclusively contained in chylomicrons, and free fatty acids in patients with type $\Pi \mathrm{Ib}$ hyperlipidemia ${ }^{20)}$. The suppression effect of ezetimibe on the postprandial increase of chylomicrons is probably attributed to inhibition of chylomicron secretion in the small intestine. Chylomicrons are formed through the aggregation of cholesterol ester, TG, and apo B48 in intestinal cells. Ezetimibe was shown to reduce the expression of fatty acid transfer protein-4 (FATP-4) and suppress the absorption of saturated fatty acids ${ }^{21}$. It is possible that ezetimibe causes the depletion of cholesterol from intestinal cells and inhibits the formation of chylomicrons, thereby being protective against the accumulation of abdominal fat.

High molecular weight adiponectin did not change at all in either group in the present study. Previously, 2-week monotherapy or combination therapy with ezetimibe and/or simvastatin did not affect serum concentrations of leptin and high molecular weight adipo$\operatorname{nectin}^{22)}$.

Meta-analysis of 23 placebo-controlled studies of lipid-lowering therapy confirmed a strong correlation between the decrease of the hs-CRP level and the decrease of LDL-C ${ }^{23)}$. Combination therapy with ezetimibe $(10 \mathrm{mg} /$ day $)$ and simvastatin resulted in a more than 2-fold decrease of hs-CRP compared to simvastatin monotherapy ${ }^{24)}$. The present study failed to find such an effect of ezetimibe in combination with pravastatin on hs-CRP.

With regard to safety, there were no serious adverse events possibly or probably related to the study drugs. Although there were no significant changes in fasting glucose and $\mathrm{HbAlc}$ in either group, fasting serum insulin and HOMA-IR increased significantly after 12 weeks to almost the same extent in both groups. The latter findings were rather unexpected because pravastatin possibly exerts a protective, rather than adverse, effect on insulin resistance ${ }^{25)}$ and glucose metabolism ${ }^{26)}$. 
Table 4. Baseline values and percent changes of non-cholesterol sterols after 12 -week treatment

\begin{tabular}{|c|c|c|c|c|c|c|}
\hline \multirow[b]{2}{*}{ Sterol $(\mu \mathrm{g} / \mathrm{mL})$} & \multicolumn{3}{|c|}{ Baseline value } & \multicolumn{3}{|c|}{ Percent change } \\
\hline & $\begin{array}{l}\text { Pravastatin }+ \text { ezetimibe } \\
\qquad(n=94)\end{array}$ & $\begin{array}{l}\text { Double-dose pravastatin } \\
\qquad(n=95)\end{array}$ & $p$ value & $\begin{array}{l}\text { Pravastatin + ezetimibe } \\
\qquad(n=94)\end{array}$ & $\begin{array}{l}\text { Double-dose pravastatin } \\
\qquad(n=95)\end{array}$ & $p$ value \\
\hline Lathosterol & $1.6(0.8)$ & $1.5(1.0)$ & 0.14 & $57.0(92.1)^{\dagger}$ & $20.9(87.2)$ & $<0.0001$ \\
\hline Campesterol & $4.0(2.4)$ & $3.6(1.7)$ & 0.36 & $-52.9(24.3)^{\dagger}$ & $13.7(42.6)^{*}$ & $<0.0001$ \\
\hline Sitosterol & $2.7(1.4)$ & $2.6(1.1)$ & 0.44 & $-42.7(25.5)^{\dagger}$ & $10.8(42.5)$ & $<0.0001$ \\
\hline $\begin{array}{l}\text { Cholestanol } \\
(\text { Ratio to TC } \times 10000)\end{array}$ & $3.0(0.9)$ & $2.8(0.8)$ & 0.13 & $-20.3(17.7)^{\dagger}$ & $2.1(26.0)$ & $<0.0001$ \\
\hline Lathosterol/TC & $7.0(3.5)$ & $6.6(4.4)$ & 0.36 & $76.0(97.9)^{\dagger}$ & $24.2(77.1)^{*}$ & $<0.0001$ \\
\hline Campesterol/TC & $17.2(9.3)$ & $16.4(7.1)$ & 0.68 & $-48.0(22.9)^{\dagger}$ & $17.0(34.8)^{\dagger}$ & $<0.0001$ \\
\hline Sitosterol/TC & $11.9(5.4)$ & $11.7(4.8)$ & 0.95 & $-36.3(24.9)^{\dagger}$ & $14.1(35.1)^{\dagger}$ & $<0.0001$ \\
\hline Cholestanol/TC & $13.2(3.6)$ & $13.0(3.2)$ & 0.66 & $-10.0(18.4)^{\dagger}$ & $6.0(22.8)^{*}$ & $<0.0001$ \\
\hline
\end{tabular}

Values are the means (SD).

${ }_{p} p<0.05$ as compared with the baseline value.

${ }_{p}^{t}<0.0001$ as compared with the baseline value.

\section{Conclusion}

Among patients under low-dose pravastatin treatment who had high LDL-C levels, add-on ezetimibe decreased LDL-C and apo B levels more markedly than a double dose of pravastatin. Add-on ezetimibe increased cholesterol synthesis, but resulted in substantial decreases in cholesterol absorption markers. Add-on ezetimibe should be the treatment of choice when statin treatment has not attained a sufficient reduction in LDL-C.

\section{Acknowledgments}

This study was funded by a clinical research grant from the International University of Health and Welfare, Tochigi, Japan

The study's Executive Committee consisted of Dr. M. Ageta, Ageta Clinic, Miyazaki; Dr. Y. Ikeda, Tagawa Municipal Hospital, Fukuoka; Dr. T. Otonari, Otonari Clinic, Fukuoka; Dr. K. Kajiwara, Jinnouchi Hospital, Kumamoto; Dr. T. Kuribayashi, Koga General Hospital, Miyazaki; Dr. T. Saikawa, Oita University, Oita; Dr. S. Hata, NHO Ureshino Medical Center, Saga; Dr. S. Biro, Tsukasa Health Care Hospital, Kagoshima; Dr. T. Sawayama, Kyushu University, Fukuoka; Dr. J. Sasaki International University of Public Health and Welfare, Fukuoka. (Principal investigator, member of protocol Committee, data interpretation and drafting of the manuscript) The Safety Monitoring Committee consisted Dr. K. Yamamoto, Takagi Hospital, Fukuoka; Dr. N. Okabe, Ayasugibiru Clinic, Fukuoka. Dr. S. Kono, Kyushu University contributed to the trial protocol, data analysis, data inter- pretation and drafting of the manuscript.

The authors thank the following physicians who participated in the study: Dr. K. Yamamoto, Dr. M. Kusuda, Dr. H. Ono, Dr. T. Shimabukuro, Dr. K. Yano, Dr. T. Taguchi, Dr. H. Tanaka, Dr. H. Kutsukake, Dr. S. Suzuki

\section{References}

1) Baigent C, Keech A, Kearney PM, Blackwell L, Buck G, Pollicino C, Kirby A, Sourjina T, Peto R, Collins R, Simes R; Cholesterol Treatment Trialists' (CTT) Collaborators: Efficacy and safety of cholesterol-lowering treatment: prospective meta-analysis of data from 90,056 participants in 14 randomised trials of statins. Lancet, 2005; 366: 12671278

2) Miettinen TA, Gylling H, Strandberg T, Sarna S: Baseline serum cholestanol as predictor of recurrent coronary events in subgroup of Scandinavian simvastatin survival study. Finnish 4S Investigators. BMJ, 1998; 316: 1127-1130

3) Strandberg TE, Tilvis RS, Pitkala KH, Miettinen TA: Cholesterol and glucose metabolism and recurrent cardiovascular events among the elderly: a prospective study. J Am Coll Cardiol, 2006; 48: 708-714

4) Miettinen TA, Gylling H, Lindbohm N, Miettinen TE, Rajaratnam RA, Relas H; Finnish Treat-to-Target Study Investigators: Serum noncholesterol sterols during inhibition of cholesterol synthesis by statins. J Lab Clin Med, 2003; 141: 131-137

5) Pisciotta L, Fasano T, Bellocchio A, Bocchi L, Sallo R, Fresa R, Colangeli I, Cantafora A, Calandra S, Bertolini S: Effect of ezetimibe coadministered with statins in genotype-confirmed heterozygous FH patients. Atherosclerosis, 2007; 194: e116-e122

6) Teramoto T, Sasaki J, Ueshima H, Egusa G, Kinoshita M, Shimamoto K, Daida H, Biro S, Hirobe K, Funahashi T, Yokote K, Yokode M: Executive summary of Japan Ath- 
erosclerosis Society (JAS) guideline for diagnosis and prevention of atherosclerotic cardiovascular diseases for Japanese. J Atheroscler Thromb, 2007; 14: 45-50

7) Macy EM, Hayes TE, Tracy RP: Variability in the measurement of C-reactive protein in healthy subjects: implications for reference intervals and epidemiological applications. Clin Chem, 1997; 43: 52-58

8) Gagné C, Bays HE, Weiss SR, Mata P, Quinto K, Melino M, Cho M, Musliner TA, Gumbiner B; Ezetimibe Study Group: Efficacy and safety of ezetimibe added to ongoing statin therapy for treatment of patients with primary hypercholesterolemia. Am J Cardiol, 2002; 90: 1084-1091

9) Pearson T, Denke M, McBride P, Battisti WP, Brady WE, Palmisano J: Effectiveness of the addition of ezetimibe to ongoing statin therapy in modifying lipid profiles and attaining low-density lipoprotein cholesterol goals in older and elderly patients: subanalyses of data from a randomized, double-blind, placebo-controlled trial. Am J Geriatr Pharmacother, 2005; 3: 218-228

10) Masana L, Mata P, Gagné C, Sirah W, Cho M, JohnsonLevonas AO, Meehan A, Troxell JK, Gumbiner B; Ezetimibe Study Group: Long-term safety and, tolerability profiles and lipid-modifying efficacy of ezetimibe coadministered with ongoing simvastatin treatment: a multicenter, randomized, double-blind, placebo-controlled, 48-week extension study. Clin Ther, 2005; 27: 174-184

11) Pearson TA, Denke MA, McBride PE, Battisti WP, Gazzara RA, Brady WE, Palmisano J: Effectiveness of ezetimibe added to ongoing statin therapy in modifying lipid profiles and low-density lipoprotein cholesterol goal attainment in patients of different races and ethnicities: a substudy of the Ezetimibe add-on to statin for effectiveness trial. Mayo Clin Proc, 2006; 81: 1177-1185

12) Davidson MH, Ballantyne CM, Kerzner B, Melani L, Sager PT, Lipka L, Strony J, Suresh R, Veltri E; Ezetimibe Study Group: Efficacy and safety of ezetimibe coadministered with statins: randomised, placebo-controlled, blinded experience in 2382 patients with primary hypercholesterolemia. Int J Clin Pract, 2004; 58: 746-755

13) Melani L, Mills R, Hassman D, Lipetz R, Lipka L, LeBeaut A, Suresh R, Mukhopadhyay P, Veltri E; Ezetimibe Study Group: Efficacy and safety of ezetimibe coadministered with pravastatin in patients with primary hypercholesterolemia: a prospective, randomized, double-blind trial. Eur Heart J, 2003; 24: 717-728

14) Dagli N, Yavuzkir M, Karaca I: The effects of high dose pravastatin and low dose pravastatin and ezetimibe combination therapy on lipid, glucose metabolism and inflammation. Inflammation, 2007; 30: 230-235

15) Sudhop T, Lütjohann D, Kodal A, Igel M, Tribble DL, Shah S, Perevozskaya I, von Bergmann K: Inhibition of intestinal cholesterol absorption by ezetimibe in humans. Circulation, 2002; 106: 1943-1948
16) Tremblay AJ, Lamarche B, Hogue JC, Couture P: Effects of ezetimibe and simvastatin on apolipoprotein B metabolism in males with mixed hyperlipidemia. J Lipid Res, 2009; 50: 1463-1471

17) McKenney JM: Optimizing LDL-C lowering with statins. Am J Ther, 2004; 11: 54-59

18) Cannon CP, Braunwald E, McCabe CH, Rader DJ, Rouleau JL, Belder R, Joyal SV, Hill KA, Pfeffer MA, Skene AM; Pravastatin or Atorvastatin Evaluation and Infection Therapy-Thrombolysis in Myocardial Infarction 22 Investigators: Intensive versus moderate lipid lowering with statins after acute coronary syndromes. N Engl J Med, 2004; 350: 1495-1504. Erratum in: N Engl J Med, 2006; 354: 778

19) Nomura M, Ishii H, Kawakami A, Yoshida M: Inhibition of Hepatic Neiman-Pick C1-Like 1 Improves Hepatic Insulin Resistance. Am J Physiol Endocrinol Metab, 2009; 297: E1030-E1038

20) Masuda D, Nakagawa-Toyama $Y$, Nakatani K, Inagaki M, Tsubakio-Yamamoto K, Sandoval JC, Ohama T, Nishida M, Ishigami M, Yamashita S: Ezetimibe improves postprandial hyperlipidaemia in patients with type $\Pi$ b hyperlipidaemia. Eur J Clin Invest, 2009; 39: 689-698

21) Sandoval JC, Nakagawa-Toyama Y, Masuda D, Tochino Y, Nakaoka H, Kawase R, Yuasa-Kawase M, Nakatani K, Inagaki M, Tsubakio-Yamamoto K, Ohama T, Matsuyama A, Nishida M, Ishigami M, Komuro I, Yamashita S: Molecular mechanisms of ezetimibe-induced attenuation of postpravastatinndial hypertriglyceridemia. J Atheroscler Thromb, 2010; 17: 914-924

22) Gouni-Berthold I, Berthold HK, Chamberland JP, Krone W, Mantzoros CS: Short-term treatment with ezetimibe, simvastatin or their combination does not alter circulating adiponectin, resistin or leptin levels in healthy men. Clin Endocrinol (Oxf), 2008; 68: 536-541

23) Kinlay S: Low-density lipoprotein-dependent and -independent effects of cholesterol-lowering therapies on Creactive protein: a meta-analysis. J Am Coll Cardiol, 2007; 49: 2003-2009

24) Sager PT, Capece R, Lipka L, Strony J, Yang B, Suresh R, Mitchel Y, Veltri E: Effects of ezetimibe coadministered with simvastatin on C-reactive protein in a large cohort of hypercholesterolemic patients. Atherosclerosis, 2005; 179: 361-367

25) Baker WL, Talati R, White CM, Coleman CI: Differing effect of statins on insulin sensitivity in non-diabetics: a systematic review and meta-analysis. Diabetes Res Clin Pract, 2010; 87: 98-107

26) Coleman CI, Reinhart K, Kluger J, White CM: The effect of statins on the development of new-onset type 2 diabetes: a meta-analysis of randomized controlled trials. Curr Med Res Opin, 2008; 24: 1359-1362 